\title{
Features predictive of MEN1 in patients with primary hyperparathyroidism
}

MEN1 mutation analysis should be undertaken for all patients with apparently sporadic primary hyperparathyroidism who are under the age of 50 years and have parathyroid hormone levels within the normal range, according to a study published in the Journal of Bone and Mineral Metabolism.

Primary hyperparathyroidism is an early expression of multiple endocrine neoplasia type 1 (MEN1), but a lack of other symptoms or a family history can hamper diagnosis of the syndrome in some patients. In addition, focal or unilateral surgical explorations for primary hyperparathyroidism can fail to identify multiglandular disease-a key indictor of MEN1related primary hyperparathyroidism rather than sporadic cases. EllerVainicher and co-investigators reasoned that identification of features highly suggestive of MEN1-related primary hyperparathyroidism would be helpful to avoid missed MEN1 diagnoses.

The 36-month, cross-sectional, observational study included 533 patients with a diagnosis of primary hyperparathyroidism from three Italian referral centers; diagnosis of sporadic or MEN1-related disease was made on the basis of the results of a range of clinical tests, and only patients with a clinical diagnosis or suspicion of MEN1-related disease underwent MEN1 mutation analysis.

The findings reveal clues to the detection of MEN1-related primary hyperparathyroidism. "Patients affected with MEN1 syndrome show more severe bone and similar renal chronic involvement in spite of a milder biochemical presentation as compared to those affected with the sporadic form", says researcher Cristina EllerVainicher of the University of Milan. Strikingly, parathyroid hormone levels in the normal range in individuals aged $<50$ years were associated with a 13.5-fold increase in risk of having MEN1. Consequently, the researchers suggest that patients with apparently sporadic primary hyperparathyroidism and these two characteristics should undergo MEN1 mutation analysis before parathyroidectomy is performed.

"In the future we would like to apply the combination 'normal parathyroid hormone levels and an age less than 50 years' as an adjunctive criterion for MEN1 analysis in order to confirm our present data", concludes Eller-Vainicher.

\section{Carol Wilson}

Original article Eller-Vainicher, C. et al. Sporadic and MEN1 related primary hyperparathyroidism: differences in clinical expression and severity. J. Bone Miner. Res. doi:10.1359/JBMR.090304 